07

\title{
Матричные фотоприемные устройства на основе слоев InSb, выращенных методом молекулярно-лучевой эпитаксии
}

\author{
() А.К. Бакаров, ${ }^{1,2}$ А.К. Гутаковский, ${ }^{1}$ К.С. Журавлев, ${ }^{1,2}$ А.П. Ковчавцев, ${ }^{1}$ А.И. Торопов, ${ }^{1}$ \\ И.Д. Бурлаков, ${ }^{3}$ К.О. Болтарь, ${ }^{3}$ П.В. Власов, ${ }^{3}$ А.А. Лопухин ${ }^{3}$ \\ ${ }^{1}$ Институт ффизики полупроводников им. А.В. Ржанова СО РАН, \\ 630090 Новосибирск, Россия \\ ${ }^{2}$ Новосибирский государственный университет, \\ 630090 Новосибирск, Россия \\ ${ }^{3}$ АО НПО „Орион“, \\ 111123 Москва, Россия \\ e-mail: bakarov@isp.nsc.ru
}

(Поступило в Редакцию 12 июля 2016 г.)

Развита технология молекулярно-лучевой эпитаксии InSb слоев на подложках InSb. На основе выращенных слоев изготовлены матричные фотоприемные устройства (МФПУ) средневолнового инфракрасного диапазона. Проведено сравнение фотоэлектрических характеристик МФПУ на основе эпитаксиальных слоев и МФПУ, серийно выпускаемых с использованием монокристаллов InSb.

DOI: 10.21883/JTF.2017.06.44514.1986

\section{Введение}

Одной из самых перспективных наукоемких технологий XXI в. является тепловидение. В настоящее время стремительно растут производство и широкое внедрение тепловизионной техники во многие отрасли хозяйственной деятельности. Без тепловизионных каналов невозможно представить современные средства наблюдения за окружающей средой и обнаружения движущихся объектов, используемые в наземных и морских условиях, в авиации и космосе. Тепловизоры активно применяются для контроля процессов промышленного производства, в астрономии, медицине, биологии и других областях. Бурное развитие тепловизионной техники потребовало кардинального совершенствования технологии ключевых элементов тепловизионной системы - инфракрасных (ИК) матричных фотоприемных устройств (МФПУ). В настоящее время наибольшее количество МФПУ средневолнового ИК-диапазона изготавливаются на основе фотодиодов из антимонида индия [1-3]. В качестве исходного материала используют монокристаллический $\mathrm{InSb}$ или эпитаксиальные слои InSb, обычно выращиваемые методом молекулярно-лучевой эпитаксии (МЛЭ). Эпитаксиальные слои менее дефектные, более однородные, имеют меньшую концентрацию фоновой примеси, чем монокристаллические пластины, что позволяет повысить характеристики МФПУ [4-7]:

- уменьшить процент дефектных элементов,

- повысить количество элементов в матрице,

- уменьшить фотоэлектрическую и динамическую взаимосвязь элементов,

- повысить рабочую температуру,

- уменьшить вес.

Кроме того, эпитаксиальное выращивание позволяет оптимизировать характеристики детекторов путем со- здания многослойных эпитаксиальных гетероструктур с заданным распределением профиля легирования и состава по толщине, что открывает возможность дальнейшего улучшения характеристик МФПУ [8].

В настоящей работе сообщается о разработке МЛЭ технологии роста InSb-слоев на проводящих подложках $\mathrm{InSb}$, представлены результаты исследования фотоэлектрических характеристик МФПУ на основе полученных слоев InSb и обсуждаются направления дальнейшего развития конструкции InSb-структур для улучшения характеристик МФПУ.

\section{Рост слоев InSb}

Слои InSb формировались методом МЛЭ в установке Compact-21T на подложках InSb (100) „epi-ready“ толщиной $500 \mu \mathrm{m}$ и диаметром $50 \mathrm{~mm}$, легированных теллуром с концентрацией электронов $(n) n=(1-3) \cdot 10^{17} \mathrm{~cm}^{-3}$. Калибровка потоков материалов осуществлялась по ионизационному вакуумметру, помещаемому во время измерения перед подложкой, и осцилляциям зеркального пучка в дифракции быстрых электронов (ДБЭ) на отражение. В качестве реперной точки для определения температуры подложки использовалась температура перехода реконструкции $c(4 \times 4) \rightarrow(1 \times 3)$ поверхности InSb (100) в потоке сурьмы [9].

На исходной поверхности подложки InSb присутствовал плотный слой окислов сурьмы и индия - после помещения подложки в ростовую камеру картинка ДБЭ представляла собой практически ровный фон. Окисел удалялся с помощью нагрева подложки в ростовой камере. Первая стадия нагрева - до появления первых признаков слета окисла - проводилась без потока сурьмы. Дальнейший нагрев подложки проводился в потоке 

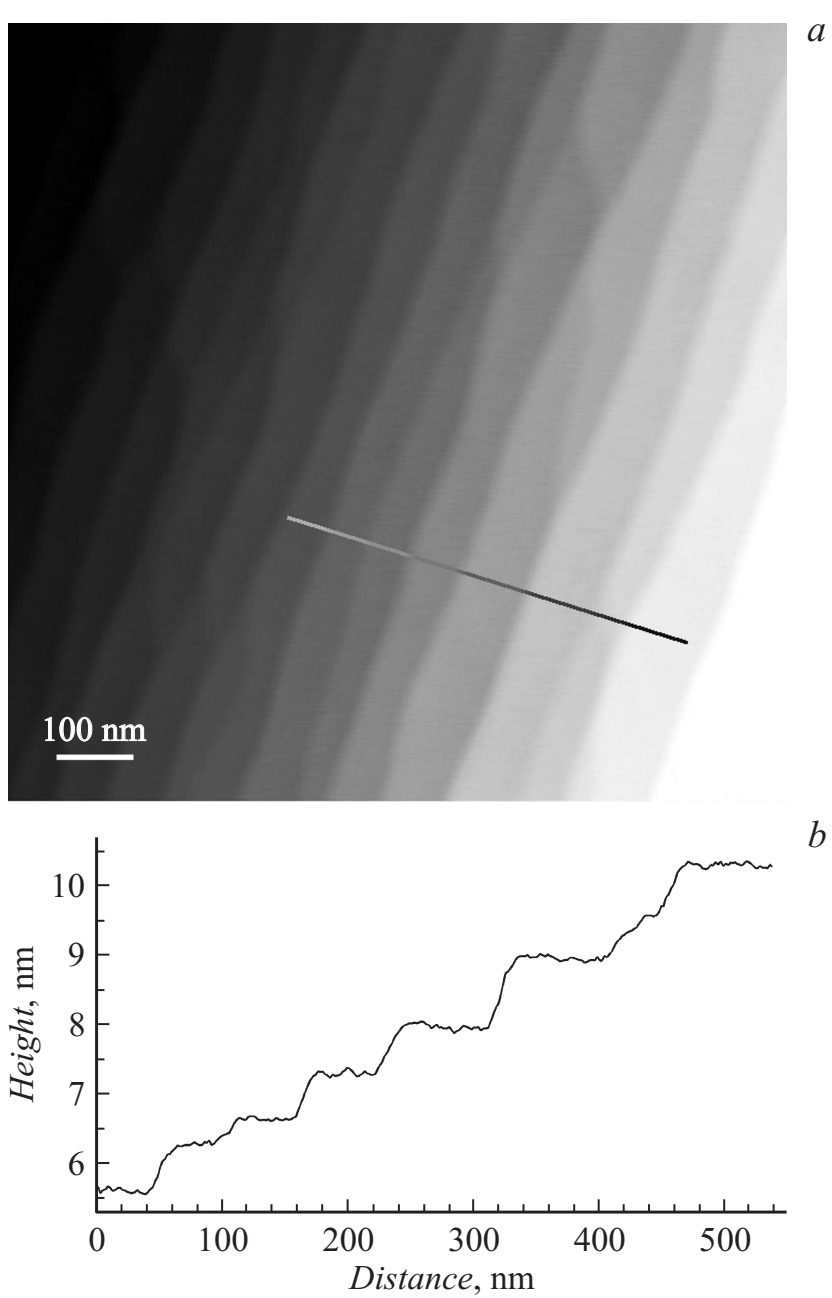

Рис. 1. $a-\mathrm{ACM}$ изображение поверхности слоя InSb в области сканирования $1 \times 1 \mu \mathrm{m}^{2}, b-$ профиль высоты вдоль линии, показанной на верхнем рисунке.

сурьмы для предотвращения ее неравновесного испарения. В результате такого отжига окисел с поверхности слетал - величина фона в картине ДБЭ значительно уменьшилась, появлялись яркие структурные рефлексы. Затем подложка охлаждалась до температуры $\sim 350^{\circ} \mathrm{C}$, и при этой температуре начинался процесс роста эпитаксиального слоя InSb. После формирования эпитаксиального слоя стали видны дополнительные, дробные рефлексы, соответствующие сверхструктуре $c(4 \times 4)$ поверхности InSb (100).

С целью оптимизации параметров роста были выращены слои InSb в условиях существования разных поверхностных сверхструктур. Измерения с помощью атомно-силового микроскопа (ACM) поверхности эпитаксиальной пленки InSb толщиной $2 \mu \mathrm{m}$, выращенной в условиях существования сверхструктуры $c(4 \times 4)$, дали значение среднеквадратичной шероховатости $(R m s)$ $R m s=2.76 \mathrm{~nm}$ (для области сканирования $20 \times 20 \mu \mathrm{m}^{2}$ ). $\mathrm{InSb}$ той же толщиной $2 \mu \mathrm{m}$, выращенный в условиях существования сверхструктуры $(1 \times 3)$, показал значе- ние $R m s=0.24 \mathrm{~nm}$. На поверхности этого слоя формируются ступени высотой $\sim 0.65 \mathrm{~nm}$ (рис. 1) и шириной $\sim 65-70 \mathrm{~nm}$, что говорит о ее высоком совершенстве.

Рост эпитаксиального слоя InSb проводился в условиях обогащения поверхности сурьмой в области существования сверхструктуры $(1 \times 3)$ при соотношении потоков сурьмы и индия $1.4: 1$ и температурах подложки $395-410^{\circ}$ С. Для создания МФПУ были выращены слои $\mathrm{InSb}$ толщиной $5 \mu \mathrm{m}$.

\section{Характеризация слоев InSb}

Структура слоев и границ раздела исследовалась методом высокоразрешающей электронной микроскопии (BРЭМ) на микроскопе JEOL-4000ЕХ при ускоряющем напряжении $400 \mathrm{kB}$. Образцы препарировались в виде поперечных срезов по плоскостям (110) по стандартной технологии, включающей шлифовку, полировку и на заключительной стадии - малоугловое травление ионами аргона с энергией $1-5 \mathrm{keV}$. Цифровая обработка экспериментальных ВРЭМ изображений проводилась с использованием пакета программ Digital Micrograph (Gatan).

Исследования методом ВРЭМ не обнаружили какихлибо дефектов структуры дислокационного типа в объеме всех изученных эпитаксиальных пленок, т.е. плотность линейных и планарных дефектов структуры была
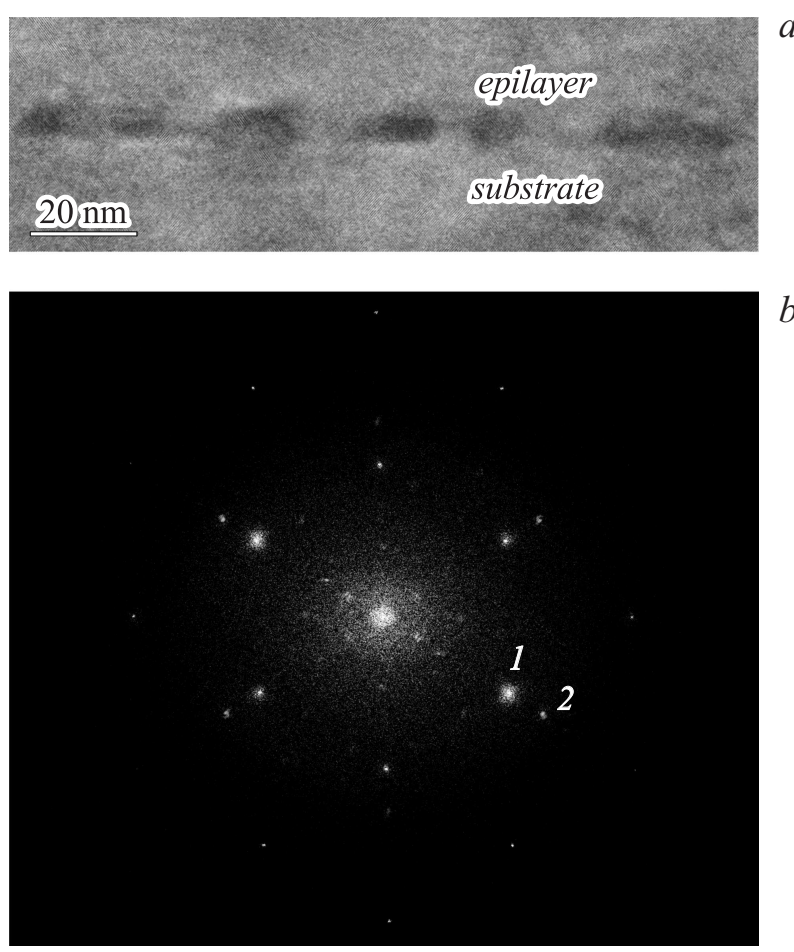

Рис. 2. $a-$ ВРЭМ изображения границы раздела эпитаксиальный слой-подложка; $b$ - картина микродифракции нанокластера $\mathrm{In}_{2} \mathrm{O}_{3}$ на гетерогранице эпитаксиальный слой $\mathrm{InSb}-$ подложка InSb (100). 1 - рефлексы InSb, $2-\mathrm{In}_{2} \mathrm{O}_{3}$. 
Сравнение параметров МФПУ на основе эпитаксиально выращенного и объемного InSb

\begin{tabular}{|c|c|c|}
\hline Наименование параметра, единицы измерения & МФПУ на эпитаксиальном InSb & МФПУ на объемном InSb \\
\hline $\begin{array}{l}\text { Время накопления, соответствующее уровню } 0.7 \\
\text { от насыщения сигнала (при относительном отверстии } \\
\text { охлаждаемой диафрагмы } 1: 1), \mathrm{ms}\end{array}$ & 1.46 & 0.54 \\
\hline Среднее значение ЭШРТ, mK & 10.5 & 11.4 \\
\hline Вольтовая чувствительность $\left(S u_{\lambda} \max \right), \mathrm{V} / \mathrm{Wt}$ & $4.8 \cdot 10^{8}\left(\mathrm{~T}_{\mathrm{H}}=0.35 \mathrm{~ms}\right)$ & $6.7 \cdot 10^{8}\left(\mathrm{TH}_{\mathrm{H}}=0.33 \mathrm{~ms}\right)$ \\
\hline Разброс чувствительности $(S t d /\langle S u\rangle), \%$ & 6.9 & 3.9 \\
\hline Количество дефектных элементов, \% & 0.12 & 0.24 \\
\hline Максимальный кластер, элементы & 3 & 15 \\
\hline $\begin{array}{l}\text { Длинноволновая граница спектральной чувствительности } \\
\text { по уровню } 0.5, \mu \mathrm{m}\end{array}$ & 4.92 & 4.98 \\
\hline $\begin{array}{l}\text { Коротковолновая граница спектральной чувствительности } \\
\text { по уровню } 0.5, \mu \mathrm{m}\end{array}$ & 4.32 & 2.93 \\
\hline Квантовый выход, \% & 56 & 85 \\
\hline Коэффициент фотоэлектрической взаимосвязи, \% & 9 & 13 \\
\hline Минимально различимая разница температур (14 штрих/мм), mK & 100 & 240 \\
\hline Темновой ток, пА & 24.5 & 8 \\
\hline Динамическая взаимосвязь, \% & 1.9 & 0.8 \\
\hline
\end{tabular}

менее $10^{4} \mathrm{~cm}^{-2}$. В то же время на достаточно толстых участках препарированного для исследований образца, когда толщина фольги более $100 \mathrm{~nm}$, на границе раздела пленка-подложка отчетливо визуализировались более темные относительно общего фона области с контрастом, не позволяющим разрешить тонкую структуру этих образований (рис. 2). Размер этих областей варьируется в диапазоне $10-30 \mathrm{~nm}$. При изучении более тонких участков препарированных объектов, когда толщина фольги составляет $20-40 \mathrm{~nm}$, удалось более детально исследовать структуру этих кластеров. На рис. $2, b$ приведены результаты такого анализа. Анализ ВРЭМ изображений и картин микродифракции показал, что на границе раздела эпитаксиальный слой $\mathrm{InSb}$ - подложка $\mathrm{InSb}$ (100) наблюдаются нанокристаллы оксида индия, эпитаксиально ориентированные относительно кристаллической решетки подложки. Рефлексы, отмеченные цифрой 2 на рис. $2, b$, соответствуют дифракции на следующих плоскостях кристаллической решетки $\mathrm{In}_{2} \mathrm{O}_{3}$, имеющей пространственную группу Іа3 (206): (222) $0.292 \mathrm{~nm} ;(400)-0.253 \mathrm{~nm}$ и $(440)-0.179 \mathrm{~nm}$.

На эпитаксиальных слоях InSb были изготовлены тестовые МДП структуры для проведения измерений вольт-фарадных характеристик с целью определения концентрации носителей заряда. Анализ $C-V$ характеристик позволил установить, что слои имеют $n$-тип проводимости с концентрацией электронов при температуре жидкого азота $n=(1.2-1.8) \cdot 10^{14} \mathrm{~cm}^{-3}$.

\section{Фотоэлектрические характеристики МФПУ}

На основе выращенных слоев были изготовлены МФПУ и проведены измерения характеристик относительной спектральной чувствительности, вольтамперной характеристики структуры „фотодиод-согласующий транзистор“, коэффициента фотоэлектрической взаимосвязи, динамической взаимосвязи („эффект памяти““) и долговременной стабильности [10]. Получены зависимости сигнала, шума и среднего значения эквивалентной шуму разности температур (ЭШРТ) МФПУ от времени накопления, значения дефектности, максимального кластера, квантового выхода, темнового тока и т.д.

В таблице приведены значения основных фотоэлектрических параметров МФПУ на основе эпитаксиально выращенного и объемного InSb. Для МФПУ на основе объемного InSb приводятся средние значения параметров серийно выпускаемых изделий [11]. Фотоэлектрические характеристики МФПУ были измерены при температуре $80 \mathrm{~K}$.

На рис. 3 показаны спектры пропускания подложки, матрицы фоточувствительных элементов и атмосферы. Видно, что степень легирования подложки $\left(n=(1.9-3.1) \cdot 10^{17} \mathrm{~cm}^{-3}\right)$ подобрана так, что коротковолновая граница ее спектра пропускания при $T=80 \mathrm{~K}$ составляет $4.75 \mu \mathrm{m}$ по уровню 0.5 . При утонении подложки до $12 \mu \mathrm{m}$ коротковолновая граница спектральной 


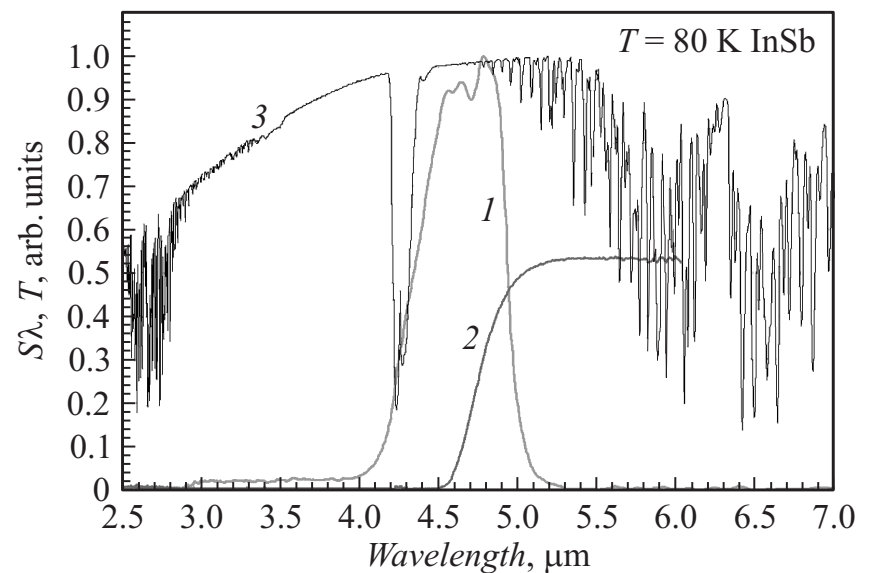

Рис. 3. Спектры пропускания матрицы фоточувствительных элементов (1), подложки (2) и атмосферы (3).

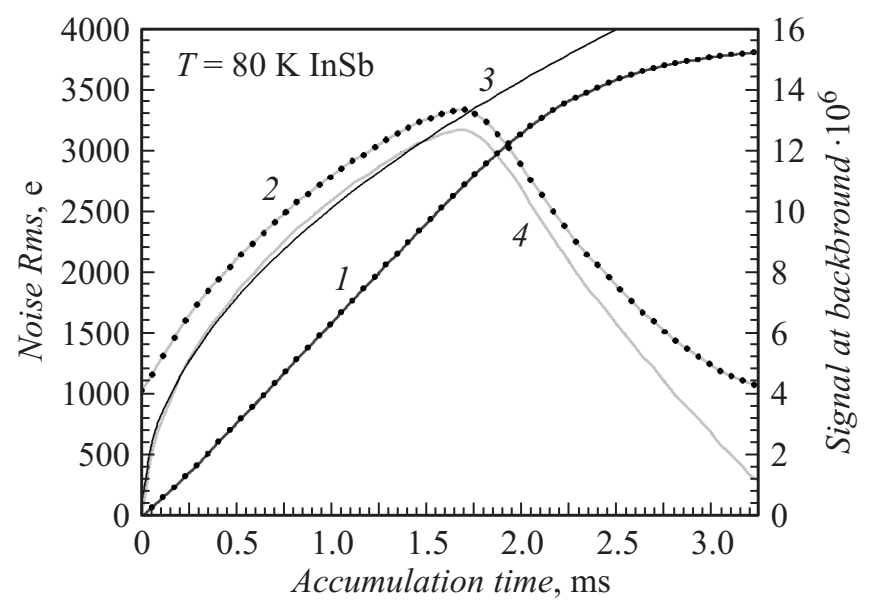

Рис. 4. Зависимости среднего значения сигнала МФПУ (1) и среднеквадратичного шума МФПУ на основе эпитаксиального $\mathrm{InSb}(2)$, расчетного шума в BLIP-режиме (3) и шума фотодиодов, полученного при вычитании из шума МФПУ шума электроники (4) от времени накопления.

чувствительности МФПУ сдвигается до $4.35 \mu \mathrm{m}$ и попадает непосредственно в окно пропускания атмосферы, гранича с линией поглощения $\mathrm{CO}_{2}(4.2 \mu \mathrm{m})$. Длинноволновая граница спектральной чувствительности МФПУ определяется охлаждаемым оптическим фильтром.

На рис. 4 представлены зависимости среднего значения сигнала МФПУ $(I)$ и среднеквадратичного шума МФПУ на основе эпитаксиального InSb (2), расчетного шума в режиме, ограниченном флуктуациями фонового излучения (BLIP-режиме) (3), и шума фотодиодов, полученного при вычитании из шума МФПУ шума электроники (4), от времени накопления. На накопительном конденсаторе в ячейке шум МФПУ при времени накопления, равном нулю, составляет $\sim 1000$ электронов. При извлечении квадратного корня из разности квадратов шума МФПУ и шума электроники видно хорошее совпадение вычтенного шума с расчетным шумом, ограниченным флуктуациями фонового излучения, что свидетельствует об отсутствии избыточных собственных шумов фотодиодов и некоррелированности шума фотодиодов и электроники считывания.

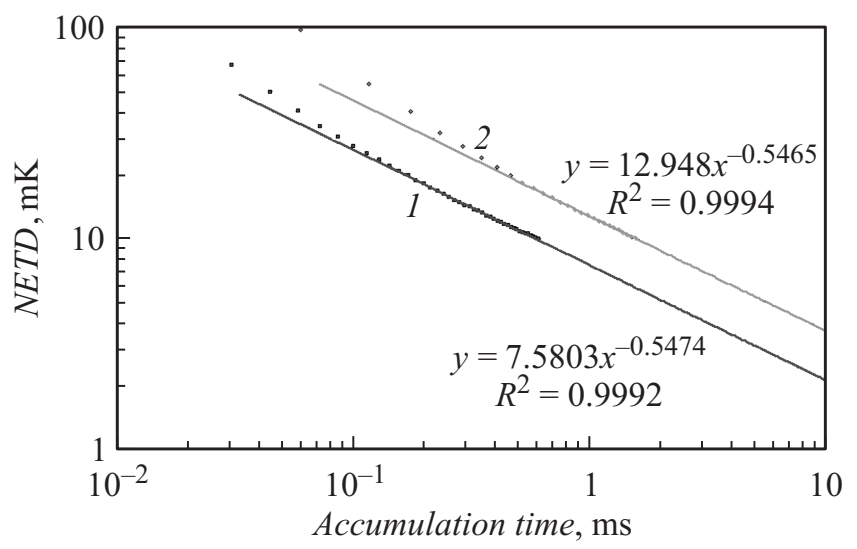

Рис. 5. Зависимость ЭШРТ от времени накопления для МФПУ на (1) эпитаксиальном и (2) объемном InSb.
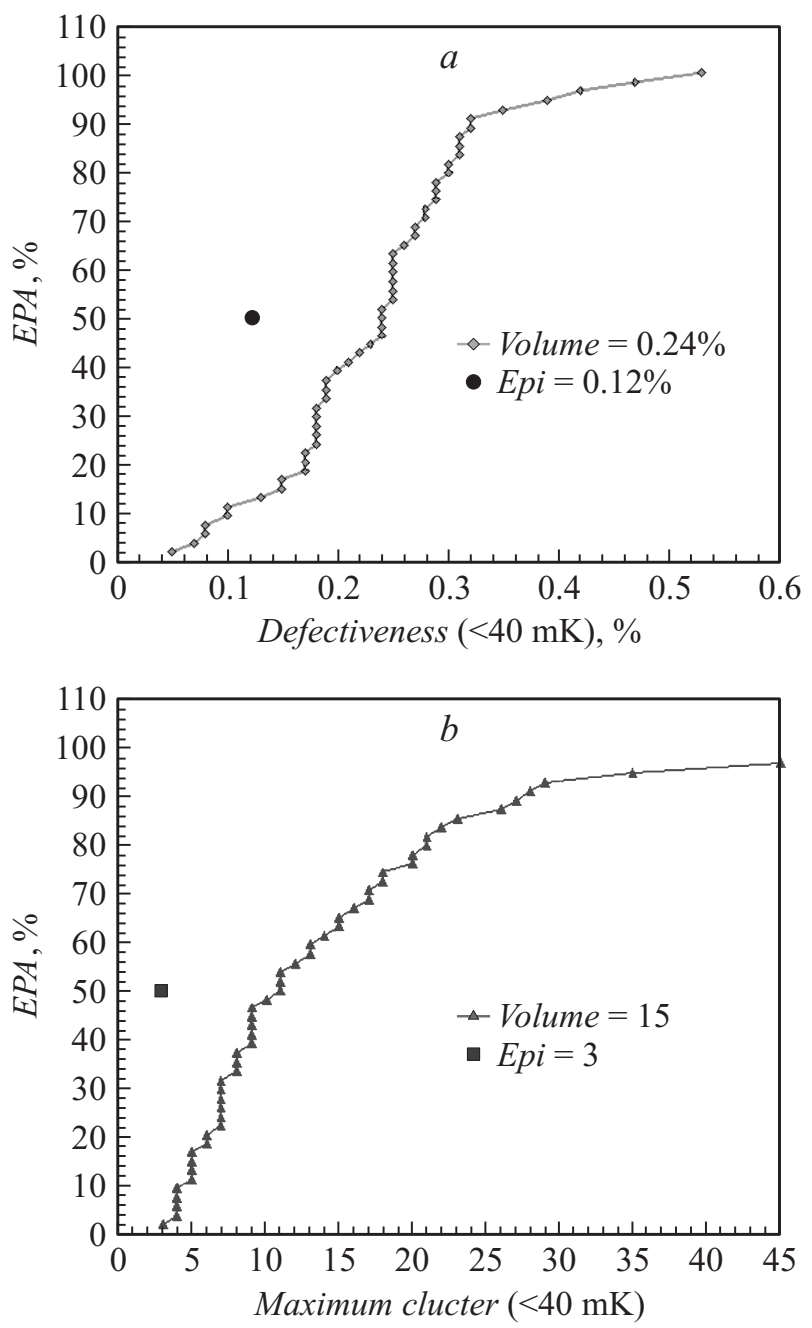

Рис. 6. Дефектность $(a)$ и максимальный кластер $(b)$ образцов МФПУ на эпитаксиальных слоях InSb (отдельные знаки) и на объемном InSb (соединенные знаки). 
На рис. 5 представлены зависимости ЭШРТ от времени накопления для МФПУ на объемном (1) и эпитаксиальном (2) InSb. Видно, что для обоих типов МФПУ в диапазоне рабочих времен накопления наблюдается близкая к корневой зависимость. Это также свидетельствует о работе МФПУ в режиме, близком к режиму ограничения фоном. Среднее значение ЭШРТ при относительном отверстии 1:0.94 на кадровой частоте $100 \mathrm{~Hz}$ при времени накопления $1.5 \mathrm{~ms}$ МФПУ на основе эпитаксиального $\mathrm{InSb}$ составило $10.5 \mathrm{mK}$, что совпадает с ЭШРТ МФПУ на основе объемного InSb при времени накопления $0.6 \mathrm{~ms}$.

Вольтовая чувствительность МФПУ на эпитаксиальном InSb оказалась меньше, что обусловлено неоптимальной толщиной фоточувствительного слоя и ограничением коротковолновой границы фоточувствительности $4.3 \mu \mathrm{m}$ из-за поглощения в подложке. Как показано на рис. 6, дефектность лучших образцов МФПУ на эпитаксиальных слоях составила $0.12 \%$, что меньше средней дефектности МФПУ на объемном InSb. Аналогично максимальный кластер оказался меньше для МФПУ с эпитаксиальным InSb. Корректное сравнение дефектности МФПУ на эпитаксиальном и объемном $\mathrm{InSb}$ возможно при увеличении выпуска МФПУ на эпитаксиальном InSb.

Для уменьшения дефектности и темновых токов, повышения однородности характеристик необходимо совершенствовать технологию подготовки подложки перед эпитаксией и качество пассивации поверхности мезаструктур, осуществлять формирование $p-n$-переходов в процессе роста. Для полного устранения взаимосвязи между элементами, обусловленной боковой диффузией неосновных носителей заряда, необходимо полное разделение элементов эпитаксиальных структур до подложки.

\section{Заключение}

В работе разработана МЛЭ технология получения слоев InSb на подложках InSb c электронным типом проводимости. На границе с подложкой обнаружены кристаллические кластеры оксида индия размером 10-30 nm. Эпитаксиальные слои InSb имеют гладкую поверхность с среднеквадратичной шероховатостью $0.24 \mathrm{~nm}$, плотность дислокаций в слоях не превышает $10^{4} \mathrm{~cm}^{-2}$, концентрация электронов при $77 \mathrm{~K}$ равна $n=(1.2-1.8) \cdot 10^{14} \mathrm{~cm}^{-3}$. На основе выращенных слоев изготовлены МФПУ. Показано, что для изготовления МФПУ можно использовать подложки с концентрацией электронов менее $10^{18} \mathrm{~cm}^{-3}$ при их утонении до $10-15 \mu \mathrm{m}$. Исследование и сравнение характеристик МФПУ на основе эпитаксиальных слоев и МФПУ, серийно выпускаемым с использованием объемного материала, показало, что

- фоточувствительные характеристики обоих типов МФПУ близки,
— квантовая эффективность МФПУ на основе эпитаксиальных слоев в максимуме спектральной чувствительности составляет $56 \%$,

- фотоэлектрическая взаимосвязь элементов МФПУ на основе эпитаксиальных слоев меньше, чем в МФПУ на основе объемного материала,

- дефектность лучших МФПУ на основе эпитаксиальных слоев меньше, чем дефектность МФПУ на объемном материале.

Исследования слоев InSb проводились на оборудовании ИФП СО РАН и ЦКП „Наноструктуры“ при поддержке Министерства образования и науки Российской Федерации (идентификатор проекта RFMEFI60414X0134).

\section{Список литературы}

[1] Rogalski A. Progr. Quant. Electron. 2012. Vol. 36. P. 342-473.

[2] $w w w$.scd.co.il SCD Semiconductor Devices. Israel.

[3] www.sbfp.com Lockheed Martin Corporation Santa Barbara Focalplane. USA.

[4] Weiguo Sun, Huitao Fan, Zhenyu Peng, Liang Zhang, Xiaolei Zhang, Lei Zhang, Zhengxiong Lu, Junjie Si, Emelyanov E., Putyato M., Semyagin B., Pchelyakov O., Preobrazhenskii V. // Inf. Phys. Technol. 2014. Vol. 62. P. 143.

[5] Комков О.С., Семенов А.Н., Фирсов Д.Д., Мельщер Б.Я., Соловьев А.А., Попова Т.В., Пихтин А.Н., Иванов С.В. // ФТП. 2011. Т. 45. С. 1481.

[6] Lien Tran T., Fariba Hatami, Ted Masselink W., Kunets Vas P., Salamo G.J. // J. Electron. Mater. 2008. Vol. 37. P. 1799.

[7] Michel E., Singh G., Slivken S., Besikci C., Bove P., Ferguson I., Razeghi M. // Appl. Phys. Lett. 1994. Vol. 65. P. 3338.

[8] Ting D.Z., Soibel A., Keo S.A., Rafol S.B., Mumolo J.M., Liu J.K., Hill C.J., Khoshakhlagh A., Hoglund L., Luiong E.M., Gunapala S.D. // J. Appl. Rem. Sens. 2014. Vol. 8. P. 084998-1.

[9] McConville C., Jones T., Leibsle F., Driver S., Noakes T., Schweitzer M., Richardson N. // Phys. Rev. B. 1994. Vol. 50. P. 14965.

[10] Болтарь К.О., Власов П.В., Лопухин А.А., Пономаренко В.П., Ранцан С.К., Фадеев В.В. // Прикладная физика. 2013. № 6. P. 67.

[11] Болтарь К.О., Власов П.В., Лопухин А.А., Полунеев В.В., Рябова А.А. // Успехи прикладной физики. 2013. № 6. P. 733. 